\title{
Medición y evaluación de parámetros meteorológicos para el centro de Bogotá, Colombia
}

\author{
Andrés Julián Aristizábal Cardona ${ }^{1 \star}$, Daniel Rolando Peña Wilches ${ }^{1 \star \star}$, Mónica \\ Andrea Botero Londoño ${ }^{2 \star \star \star}$ \\ 1 Departamento de Ingeniería Electrónica \\ 2 Departamento de Ciencias Naturales \\ Universidad Central \\ Bogotá, Colombia
}

FeCha De ENTREGA: MARZo 14 DE 2012

Fecha de EVAluación: ABRIL 4 DE 2012

FECHA DE APROBACión: MAYO 3 DE 2012

\begin{abstract}
Resumen Mediante el empleo de instrumentación virtual, El Grupo de Procesos y Soluciones Energéticas (GP\&SE) de la Universidad Central en Bogotá, adelanta investigaciones relacionadas con la medición y evaluación de radiación solar global, temperatura ambiente y velocidad y dirección de viento en la capital del país.

Los análisis resultantes permiten caracterizar las variables mencionadas para el diseño de sistemas fotovoltaicos debido a que el potencial de electricidad solar de un lugar depende de la temperatura y de la radiación solar de la localidad.

En este trabajo se presentan detalles del diseño del sistema de medición de los parámetros, la instrumentación electrónica implementada y las evaluaciones estadísticas del primer proyecto piloto desarrollado en el 2010.
\end{abstract}

Palabras Clave: radiación solar, viento, temperatura, instrumento virtual, sensores.

* Posdoctorado Proyecto de Distribución Eléctrica Inteligente SILICE, Universidad Nacional de Colombia, 2010. Doctorado en Ciencias Física, Universidad Nacional de Colombia, 2008. Ingeniería Eléctrica, Universidad Nacional de Colombia, 2004. Docente de Tiempo Completo - Universidad Central, 2009 - Actual. Áreas de interés: Energía Solar, Instrumentación Electrónica y Sensores, Calidad de Energía, Generación Eléctrica Distribuida. jaristizabalc@ucentral.edu.co

** Ingeniería Electrónica - Universidad Central, 2010. Áreas de interés: Energías Renovables, Instrumentación Electrónica, Automatización y Control. dpenaw@ucentral .edu.co

*** Doctorado en Ciencias Física, Universidad Nacional de Colombia, 2008. Maestría en Física, Universidad Nacional de Colombia, 2003. Especialización Materiales, Universidad Nacional de Colombia, 2002. Áreas de interés: Materiales Semiconductores, Espectofotometría, Propiedades Ópticas y Eléctricas de los materiales. mboterol@ucentral.edu.co 


\begin{abstract}
Grupo de Procesos y Soluciones Energéticas (GP\&SE) of Universidad Central in Bogotá carries out investigations related to the measurement and evaluation of global solar radiation, environmental temperature, and wind speed and direction in the capital of Colombia by using virtual instruments. The resulting analysis allows characterizing all these variables for the design of photovoltaic systems due to the fact that the solar electricity potential of a place depends on the temperature and solar radiation of the location.

This paper presents details on the measure system design of the electronic instrumentation implemented and the statistical evaluations of the first pilot project developed in 2010 .
\end{abstract}

Keywords: solar radiation, wind, temperature, virtual instrument, sensors.

\title{
1. Introducción
}

Las nuevas tecnologías han permitido encontrar un gran número de soluciones a problemas que antes eran incuestionables. De esta manera, el uso y aprovechamiento de la energía eléctrica en sus diferentes manifestaciones provee una gran cantidad de propuestas interesantes para suplir definitivamente los combustibles fósiles como gas, petróleo y carbón. La ingeniería entonces debe buscar y justificar cuál de las soluciones se adapta mejor al problema y realizar una serie de cuestionamientos para así determinar la mejor alternativa a implementar.

En el mercado mundial existen diferentes mecanismos de generación limpia capaces de entregar potenciales muy significativos de energía, aunque con un elevado costo de implementación. Es necesario realizar un estudio de campo que permita determinar la viabilidad de los recursos renovables con el fin de no caer en sobrecostos y pérdidas generadas en las distintas etapas de producción de dichos sistemas.

Es bien conocido que en la actualidad una de las grandes prioridades a escala mundial, y en particular de nuestro país, es el desarrollo de fuentes alternas de generación de energía eléctrica, y en especial de fuentes renovables que produzcan una baja contaminación ambiental. En este contexto, la generación fotovoltaica de energía eléctrica es uno de los más firmes candidatos; ya que además de existir un gran potencial solar en el país, no genera contaminación ambiental.

La adquisición de datos en sistemas de monitoreo de variables ambientales y de sistemas fotovoltaicos, en general siguen una arquitectura tradicional que emplea dataloggers o microcontroladores [1,2], con funciones como, establecer el tiempo en que se debe tomar una muestra de los sensores, transformar señales análogas de los sensores en señales digitales y almacenarlas en la memoria del computador y finalmente, controlar un módulo de comunicaciones para transmitir la información a través del puerto serial del PC (puerto RS-232); para esto, una amplia variedad de tarjetas de adquisición de datos, DAQ, han sido usadas $[3,4,5,6]$.

Aunque parece sencillo, medir parámetros físicos es bastante complicado. En primer lugar se debe establecer el tipo de variable a tratar, el sensor a instrumentar, 
el sistema de almacenamiento de datos y finalmente el tratamiento estadístico de los mismos; sin embargo en este proceso se realizan ajustes de calibración para realizar un análisis más preciso y brindar un estudio de campo lo suficientemente amplio como para cumplir el objetivo primordial de la investigación. En este punto es donde se debe verificar la veracidad de la información obtenida por los sensores, es decir: los valores corresponden a la magnitud física de la variable estudiada?, con estos datos realmente es posible dimensionar un sistema?, y finalmente, se dispone del tiempo necesario para realizar el estudio?. Estos interrogantes describen perfectamente el problema de las estaciones meteorológicas con las que contamos en la actualidad, ya que muchas de ellas no tienen los sensores adecuados, carecen de medición en tiempo real, baja precisión y un monitoreo escaso ya que se revisan con periodos de tiempo seleccionados al azar o se ubican en lugares inadecuados para tal propósito.

\section{Características de implementación}

Dentro de las primeras actividades del proyecto, se seleccionaron los sensores adecuados para la medición de cada una las variables físicas, así como el lugar de instalación de la estación de medición. Una vez analizados estos criterios y dado que se debe estimar la velocidad y dirección del viento, es conveniente instalar una torre en un lugar de al menos diez metros de altura, donde se pueda colocar este dispositivo, y además permita la incorporación a una distancia razonable del piranómetro para medir la radiación solar global y el termistor para estimar la temperatura ambiente.

Luego de este proceso es necesario recopilar o adquirir la información en una computadora para su posterior análisis. Para ello es necesario utilizar el software LabVIEW de National Instruments, como sistema de adquisición de datos mediante el desarrollo de un instrumento virtual capaz de interpretar, convertir y guardar la información de los sensores descritos anteriormente. La figura 1 presenta el diagrama de bloques general de la estación de medición.

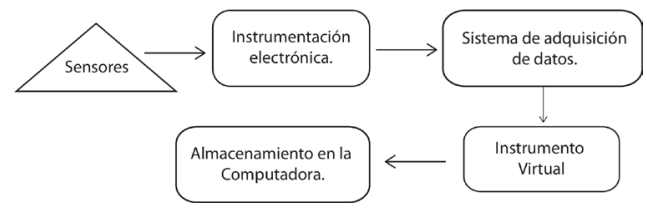

Figura 1. Esquema de funcionamiento del sistema de monitoreo.

El acondicionamiento de los sensores del sistema de medida sigue siendo tradicional, es decir que se debe seguir el proceso normal de traducción de las señales análogas de cada uno de los sensores para que los valores de tensión y corriente sean acordes a cada una de las variables físicas establecidas. 
En principio se realizó un estado del arte de dispositivos similares para determinar las necesidades técnicas para la implementación de la plataforma de estudio y el acondicionamiento de los sensores para adquirir la información. Luego de establecer dichas necesidades se asignaron las siguientes prioridades para obtener mejores resultados en el proyecto.

- Diseño físico de la estación para instalar los sensores.

- Elementos de hardware necesarios para la estación.

- Caracterización de la variable y circuitos electrónicos de acondicionamiento.

\subsection{Diseño y disposición de la estación de medición}

Dentro de los predios de la Universidad Central se ha dispuesto una terraza en la torre de la carrera de Economía, ya que al tratarse de un lugar elevado no es necesario incorporar un pedestal de gran tamaño para la instalación de los sensores del sistema. Se estimó que las dimensiones del pedestal deben ser de tres metros de altura para la colocación del anemómetro en la parte más alta, sin la necesidad de incorporar un pararrayos de protección adicional, ya que las instalaciones de la universidad cuentan con apantallamiento a tierra y los mecanismos de monitoreo también lo poseen de manera independiente.

Para brindar sostén al pedestal se diseñó una plataforma metálica de $30 \times$ $30 \mathrm{~cm}$ y $\frac{1}{2}$ pulgada de espesor con perforaciones en sus cuatro esquinas para la colocación de pernos expansivos de $\frac{1}{2} \times 3$ pulgadas y tener mayor agarre a la terraza. Además de incluir cuatro soportes laterales adicionales de $15 \times 15$ cm en platina de $\frac{3}{8}$ de pulgada para conservar la verticalidad el pedestal. En la parte superior del pedestal se han añadido cuatro platinas de dos pulgadas de ancho y $15 \mathrm{~cm}$ largo donde se colocaron los perfiles de aluminio de $40 \mathrm{~cm}$ de largo, teniendo la precaución de orientarlos en diferentes direcciones para evitar la interferencia entre sensores. La figura 2 presenta la disposición de los sensores.

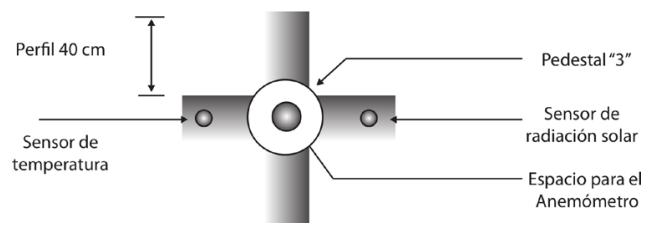

Figura 2. Soporte superior de instalación de los sensores.

En cada uno de los perfiles laterales se muestra un sensor diferente, reservando la parte superior para el anemómetro ya que se debe acoplar con una unión de $\frac{3}{4}$ de pulgada y dos agarraderas de la misma medida, esto evita que oscile por el movimiento de las cazoletas y lo mantiene separado al menos $30 \mathrm{~cm}$ de los demás sensores. 


\subsection{Sistema de adquisición de datos}

Es conveniente establecer el tipo de señal y sus características especiales como impedancia de entrada y salida, tensiones de alimentación y protecciones necesarias en los circuitos, para garantizar que las mediciones tengan la mayor precisión posible.

El instrumento de adquisición de datos seleccionado fue un Compact FieldPoint CFP 2020 de fabricación americana de la compañía National Instruments, diseñado para aplicaciones de tipo industrial en procesos de adquisición de datos y sistemas de control o PID. Tiene la posibilidad de conexión a Ethernet, RS-232, RS-485 y RJ-45 de tal manera que cumple con las características necesarias para determinar las variaciones de los sensores e interpretarlas mediante un instrumento virtual desarrollado en el software de aplicación LabVIEW [8]. El dispositivo CFP 2020 es una interface que permite la comunicación de los módulos de control conectados al chasis de expansión y la computadora, es decir que no posee canales de entrada como tal para adquirir señales de tensión o corriente. Es necesario contar con un módulo de conexiones CFP AL-110 que posee los canales necesarios para que el primer dispositivo gestione la información recibida y finalmente la procese el instrumento virtual [8]. En el siguiente cuadro de presentan las especificaciones de éste módulo.

\begin{tabular}{|c|c|}
\hline \multicolumn{2}{|c|}{ Características del Compact FieldPoint CFP AL-110 } \\
\hline Número de canales & 8 input / output. \\
\hline Resolución & 16 Bits. \\
\hline Tipo de entrada & Voltaje / Intensidad. \\
\hline Rango de las señales & $\begin{array}{l} \pm 60 \mathrm{mV}, \pm 300 \mathrm{mV}, \pm 1 \mathrm{~V}, \pm 5 \mathrm{~V}, \pm 10 \mathrm{~V} \text { y de } 0 \text { a } 10 \mathrm{~V} \\
\pm 20 \mathrm{~mA}, 200 \mathrm{~mA} \text { y de } 4 \text { a } 20 \mathrm{~mA}\end{array}$ \\
\hline Filtro de ruido $50 / 60 \mathrm{~Hz}$ & Por selección de software de $5 \mathrm{~Hz}$ a $0,66 \mathrm{~Hz}$. \\
\hline Impedancia de entrada & $100 \mathrm{M} \Omega$ \\
\hline Protección de sobrevoltaje & $40 \mathrm{~V}$ \\
\hline Temperatura de operación & $-40{ }^{\circ} \mathrm{C}$ a $70{ }^{\circ} \mathrm{C}$ \\
\hline
\end{tabular}

Cuadro 1. Especificaciones técnicas del CFP AL-110 [8].

Finalmente el chasis ensamblado, se conecta por el puerto serial RS-232 para adquirir los datos en los canales de entrada y por el puerto Ethernet para gestionar la comunicación con la computadora en tiempo real y realizar un posterior análisis con la información almacenada.

\subsection{Selección y diseño de instrumentos}

En los cuadros 2 y 3 se presentan las especificaciones técnicas de los sensores para medir las variables de interés. 


\begin{tabular}{cc}
\hline Propiedades & Piranómetro Sp-Lite \\
\hline Rango espectral & $400 \mathrm{~nm}-1100 \mathrm{~nm}$ \\
\hline Sensibilidad & $72 \mu \mathrm{V} / \mathrm{Wm}^{2}$ \\
\hline Tiempo de respuesta & $<1 \mathrm{~s}$ \\
\hline Radiación máxima & $2000 \mathrm{Wm}^{2}$ \\
\hline Dependencia de temperatura & $+0,15 \% /{ }^{\circ} \mathrm{C}$ (típica) \\
\hline Rango de temperatura & $-30{ }^{\circ} \mathrm{C}$ a $70{ }^{\circ} \mathrm{C}$ \\
\hline Error de dirección & $\pm 65 \%$ para ángulos $>80^{\circ}$ \\
\hline
\end{tabular}

Cuadro 2. Características del instrumento para medir radiación solar global.

\begin{tabular}{cc}
\hline Propiedades & Anemómetro Met One \\
\hline Exactitud & $\begin{array}{c} \pm 0,12 \mathrm{~m} / \mathrm{s} \text { para vel. de viento }<10,10 \mathrm{~m} / \mathrm{s} \\
\pm 1.1 \% \text { para vel. de viento }>10,1 \mathrm{~m} / \mathrm{s}\end{array}$ \\
\hline Umbral & $0,40 \mathrm{~m} / \mathrm{s}$ \\
\hline Rango de operación & 0 a $49 \mathrm{~m} / \mathrm{s}$ \\
\hline Señal de salida & Contacto de cerrado \\
\hline
\end{tabular}

Cuadro 3. Características del instrumento para medir velocidad y dirección del viento.

Para la medición de la temperatura, se utilizó un termistor NTC de $5 \mathrm{k} \Omega$ y se emplea una resistencia para limitar la corriente eléctrica que circula a través del mismo, garantizando la estabilidad eléctrica en su operación y la confiabilidad en las medidas de temperatura, por esto, es necesario polarizar con una tensión regulada, que en este caso es generada con un regulador LM7805.

Acondicionamiento del sensor de viento. Empleamos un anemómetro MET ONE modelo 034B de cazoletas, de tal manera que para encontrar la velocidad del viento se debe tomar el pulso de conteo por cada instrucción, con el interruptor en configuración de cierre.

Fue necesario realizar un conversor de frecuencia a voltaje debido a que la señal de salida en configuración de pulsador cerrado es una onda cuadrada; es decir que al completar una revolución, envía una señal pulso proporcional al caudal de viento de manera similar a un encoder.

Se pudo determinar que se requería utilizar un filtro pasa-bajas con una frecuencia de corte de $159 \mathrm{~Hz}$ que es el rebote de la señal producida por la fuente de alimentación de $60 \mathrm{~Hz}$ y la frecuencia máxima esperada del dispositivo, alrededor de $75 \mathrm{~Hz}$. Sin embargo, existe una desventaja y es que se añade un offset de 5 VDC a la señal de entrada por lo que debe ser reconstruida con un circuito restador como se muestra en la figura 3. 


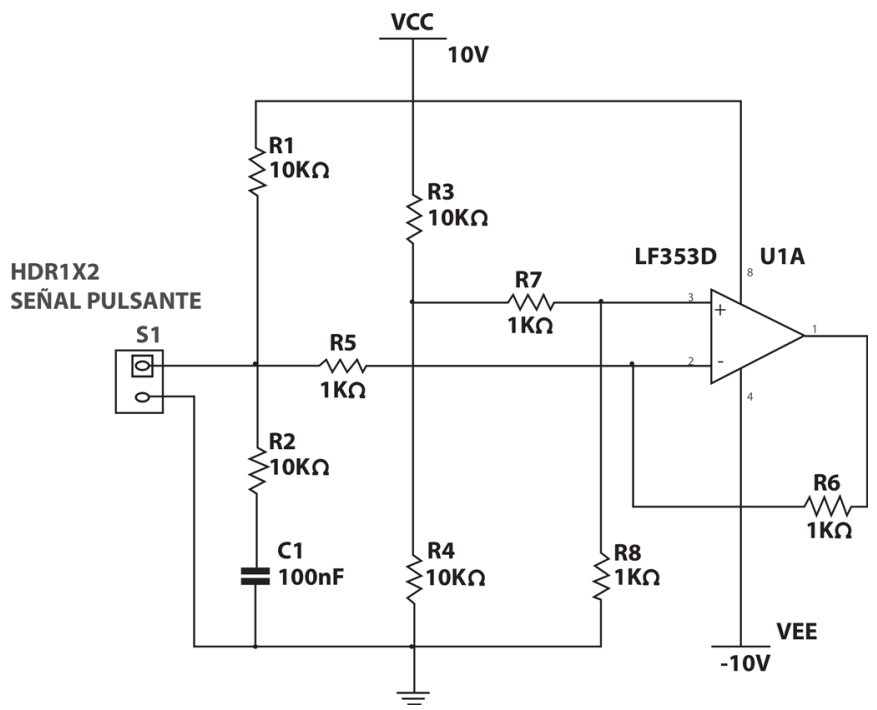

Figura 3. Filtro pasa-bajas de primer orden, con restador como reconstructor de la señal de entrada del anemómetro.

Según datos del fabricante, si se estima que la frecuencia máxima es de 74,76 $\mathrm{Hz}$, se obtiene una excitación de voltaje de $10 \mathrm{~V}$, con corriente máxima de cierre de $5 \mathrm{~mA}$ que equivalen a $60 \mathrm{~m} / \mathrm{s}$.

De esta manera se puede decir que el conversor adecuado para tener un error mínimo es el LM331, ya que dependiendo de la configuración seleccionada, puede conservar una linealidad que varía entre el $1 \%$ y el $6 \%$, dependiendo de la velocidad de respuesta y el tiempo de conversión, suministrando valores de tensión proporcionales al caudal de viento medido. Teniendo en cuenta estos aspectos se seleccionó el circuito mostrado en la figura 4.

Acondicionamiento del sensor de radiación solar global. La salida proporcional de voltaje estará dada por la relación de energía:

$$
E_{\text {solar }}=\frac{v_{\text {emf }}}{s}=\left[\frac{W}{m^{2}}\right]
$$

Después de evaluar la señal de salida, se concluyó que es necesario amplificar la señal de entrada 11 veces para que el CFP 2020 pueda estimar los cambios de radiación global.

De tal manera que la salida del amplificador no inversor es:

$$
\begin{gathered}
V_{\text {out }}=V_{\text {in }}\left(1+\frac{R_{1}}{R_{2}}\right) \\
V_{\text {out }}=V_{\text {in }}(11)
\end{gathered}
$$




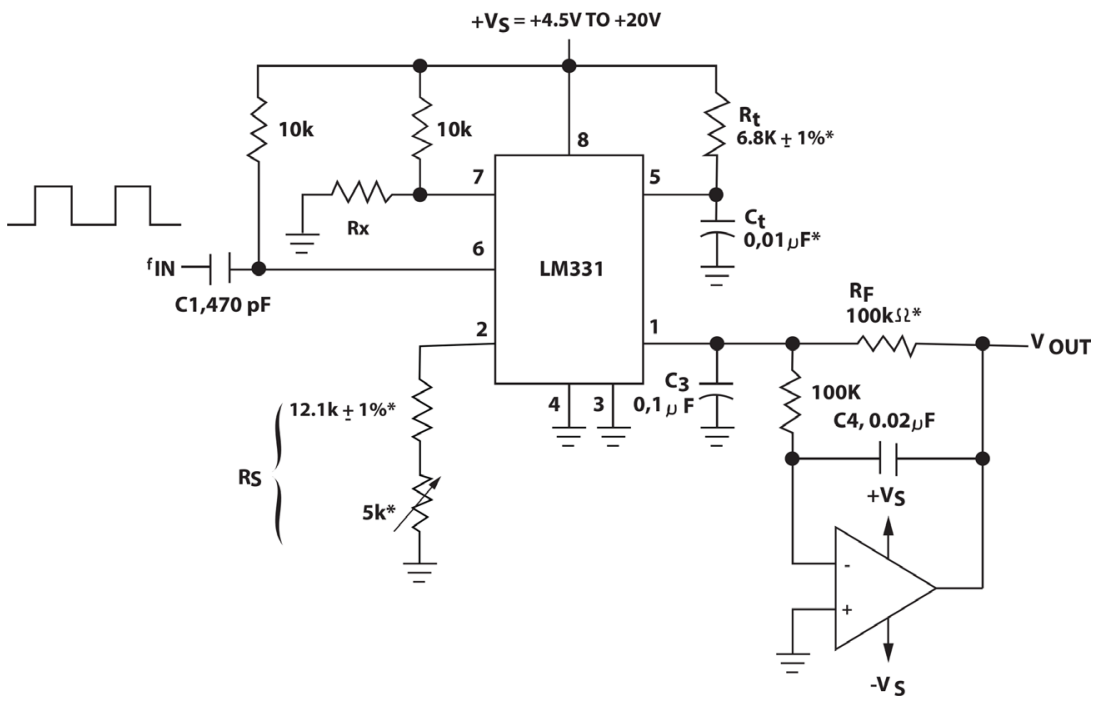

Figura 4. Conversor de frecuencia a voltaje de $0-10 \mathrm{kHz}$ de alta precisión con filtro pasa-bajas de segundo orden y $\pm 0,01 \%$ de no linealidad máxima.

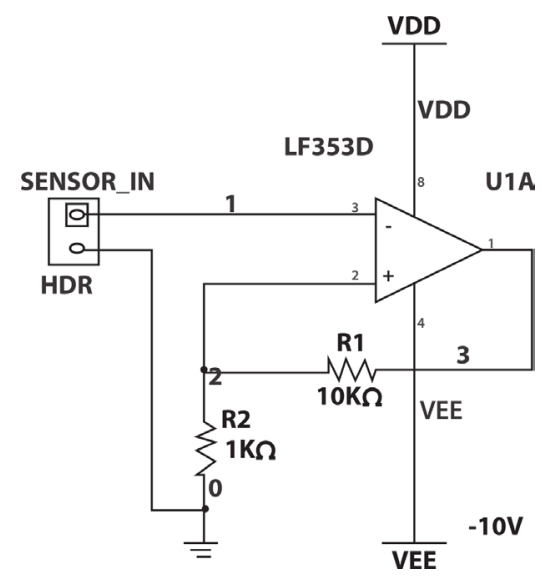

Figura 5. Configuración del amplificador no inversor para el sensor de radiación global.

Acondicionamiento del sensor de temperatura-termistor NTC. De una forma aproximada, pero no por ello lejos de lo real, la variación de la resistencia eléctrica de un metal a raíz de la temperatura puede presentarse mediante la expresión:

$$
R(t)=R_{0}(1+a t)
$$


Donde $R(t)$ : resistencia eléctrica dependiente de la temperatura es proporcional a $R_{0}$ : resistencia eléctrica a la temperatura de $0{ }^{\circ} \mathrm{C}$ y $a$ : coeficiente de variación.

En el caso práctico se emplea una NTC: termoresistencia que disminuye el valor de su coeficiente térmico conforme va aumentando la temperatura reflejando los diferentes valores de temperatura en valores de voltaje. Las termorresistencias de uso más común se fabrican de alambres finos soportados por un material aislante y luego encapsulados. El elemento encapsulado se inserta luego dentro de una vaina o tubo metálico cerrado en un extremo que se llena con un polvo aislante y se sella con cemento para impedir que absorba humedad. Los materiales utilizados para los arrollamientos son fundamentalmente Platino, Níquel, NíquelHierro, Cobre y Tungsteno.

Utilizando una resistencia $5 \mathrm{k} \Omega$ denotada en la figura 6 como $R_{1}$, se limita la corriente eléctrica que circula a través del termistor; garantizando la estabilidad eléctrica en la operación del termistor y la confiabilidad en las medidas de temperatura. Por esto, es necesario polarizar con una tensión regulada que en este caso es generada con un regulador LM7805. $R_{1}$ es una resistencia de precisión donde de la tensión resultante en el termistor es directamente proporcional a la resistencia del mismo y se emplea como señal eléctrica indirecta para la medida de la temperatura.

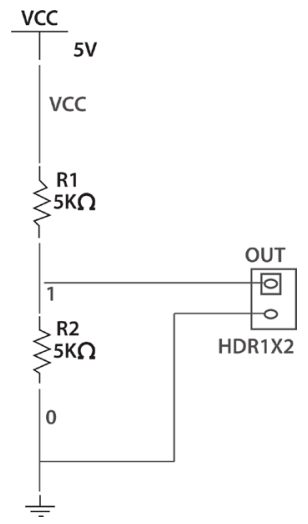

Figura 6. Configuración del divisor de voltaje donde $R_{2}$ es el termistor.

La temperatura cambia en función de patrones climáticos establecidos aunque la ubicación geográfica del lugar se encuentre cerca del Ecuador. Se ha determinado realizar mediciones de ciclos completos de 24 horas con intervalos de 2 a 5 segundos, porque las variaciones del coeficiente térmico son amplias al tener una gran sensibilidad al medio y poca histéresis. 


\subsection{Configuración del sistema de adquisición de datos y desarrollo de instrumentos virtuales}

La instrumentación virtual es una herramienta relativamente nueva en el campo de aplicación y medición de variables físicas. En el caso de Colombia, los productos de National Instruments son una innovación tecnológica que permiten flexibilizar los métodos de estimación, al incorporar interfaces electrónicas de fácil manipulación y para propósitos específicos. Para este estudio se dispone de un Compact FieldPoint CFP 2020 como interface para realizar dicha tarea, aunque por sí solo no realiza ningún tipo de análisis. El dispositivo físico debe ser gobernado por un instrumento virtual a partir de las necesidades que se han establecido en el proceso de investigación y desarrollar un modelo gráfico para lograr una interpretación clara de los resultados.

Luego de analizar las variables se encontraron tres características vitales para su proceso de estimación:

- El diseño del instrumento virtual debe proporcionar un intervalo de medición en minutos, horas y días para realizar el estudio de campo.

- Cada una de las señales eléctricas de tensión provistas por los sensores, deben ser convertidas en un valor estándar de medición para ser almacenados en la computadora en los intervalos descritos anteriormente y luego ser analizados.

- Desarrollo del software mediante instrumentación virtual para la configuración del sistema de adquisición de datos, procesamiento y almacenamiento de la información.

Empleando el software de configuración "Measurement \& Automation ExploresMAX" de National Instruments presentado en la figura 7, fue configurado el sistema CFP 2020 - CFP AL-110 y asignados los canales de adquisición de datos tal y como se expone en el cuadro 4.

\begin{tabular}{cccc}
\hline Resumen $\boldsymbol{y}$ asignación de canales & de entrada \\
\hline Canal de entrada & Variable & Vin COM \\
\hline Channel 0 & Radiación Global & 1 & 18 \\
\hline Channel 1 & Temperatura & 3 & 20 \\
\hline Channel 2 & Velocidad de viento & 5 & 22 \\
\hline Channel 3 & Dirección de viento & 7 & 24 \\
\hline
\end{tabular}

Cuadro 4. Resumen de canales asignados a cada variable.

En la figura 8 se presenta el panel frontal del VI principal, desarrollado para agrupar los diferentes sub-instrumentos virtuales (sub-VI's) que se utilizarán en la medición de las variables de interés.

Las señales así acondicionadas electrónicamente son adquiridas por el sistema Compact FieldPoint 2020 a una velocidad de 1 kS/s y con una resolución de 16 


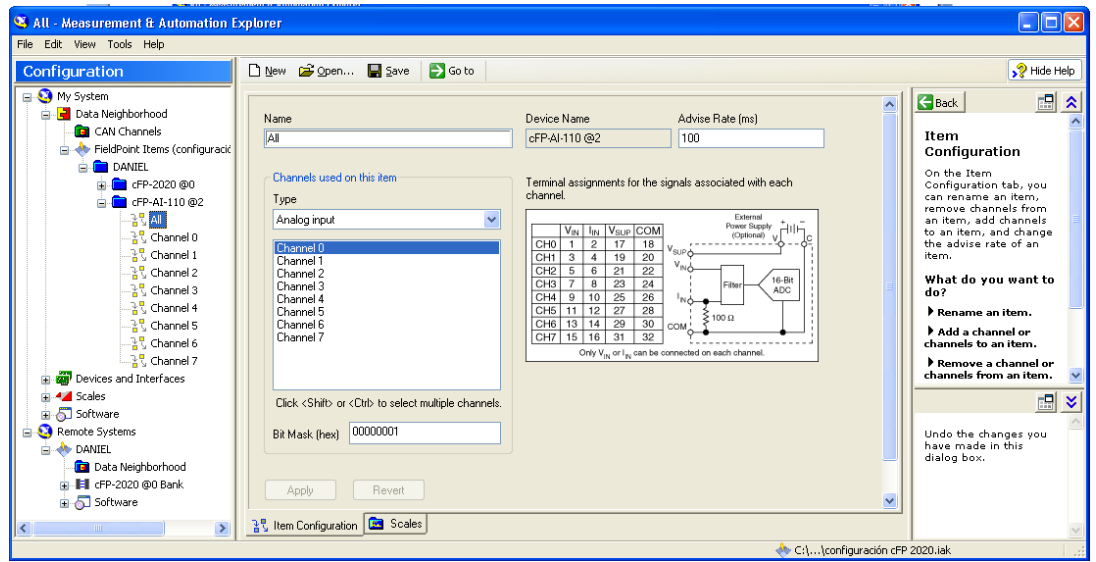

Figura 7. Configuracion de cableado I/O disponibles en el CFP AL-110.

bits. Después de la adquisición, las señales son procesadas para presentarse en el panel frontal y se almacenan en registros de minutos, horas y días en formato .xls.

\section{Resultados}

El período de estudio está comprendido entre abril de 2010 y abril de 2011 para todas las mediciones.

En la figura 9 se presenta el comportamiento de la velocidad del viento para el mes de junio de 2010 como uno de los más representativos. Se puede observar un comportamiento constante con pocos picos de incidencia salvo los presentes en los días 14 y 20 con un promedio de $3,5 \mathrm{~m} / \mathrm{s}$ y se conserva el caudal máximo de aire de $4,43 \mathrm{~m} / \mathrm{s}$ en al menos 25 de los 30 días que componen el mes. Sorprende el promedio general que es de $0,82 \mathrm{~m} / \mathrm{s}$ y el valor mínimo registrado de $0,28 \mathrm{~m} / \mathrm{s}$ como se muestra en el cuadro 5. Estos resultados son propios del fenómeno del niño y contrastan con los días soleados y lluvias leves que se presentaron para esas fechas.

En la dirección del viento se presentaron algunas variaciones importantes entre las puntos Sureste y Noroeste, siendo predominante la primera de ellas con intervalo entre $234,1^{\circ}$ y $253^{\circ}$. En cuanto a la segunda, la dirección cambia entre los $164,9^{\circ}$ y $172,4^{\circ}$ con un caudal similar en todos los casos como se muestra en la figura 10 .

El cuadro 6 expone la estadística de los datos de velocidad y dirección del viento (junio 2010) generada por el instrumento virtual encargado de la adquisición y análisis de las señales.

La dirección Sureste predomina en el mes de junio con una participación del $83.33 \%$ mientras que el noreste presento un incremento del 3,66\% llegando a 


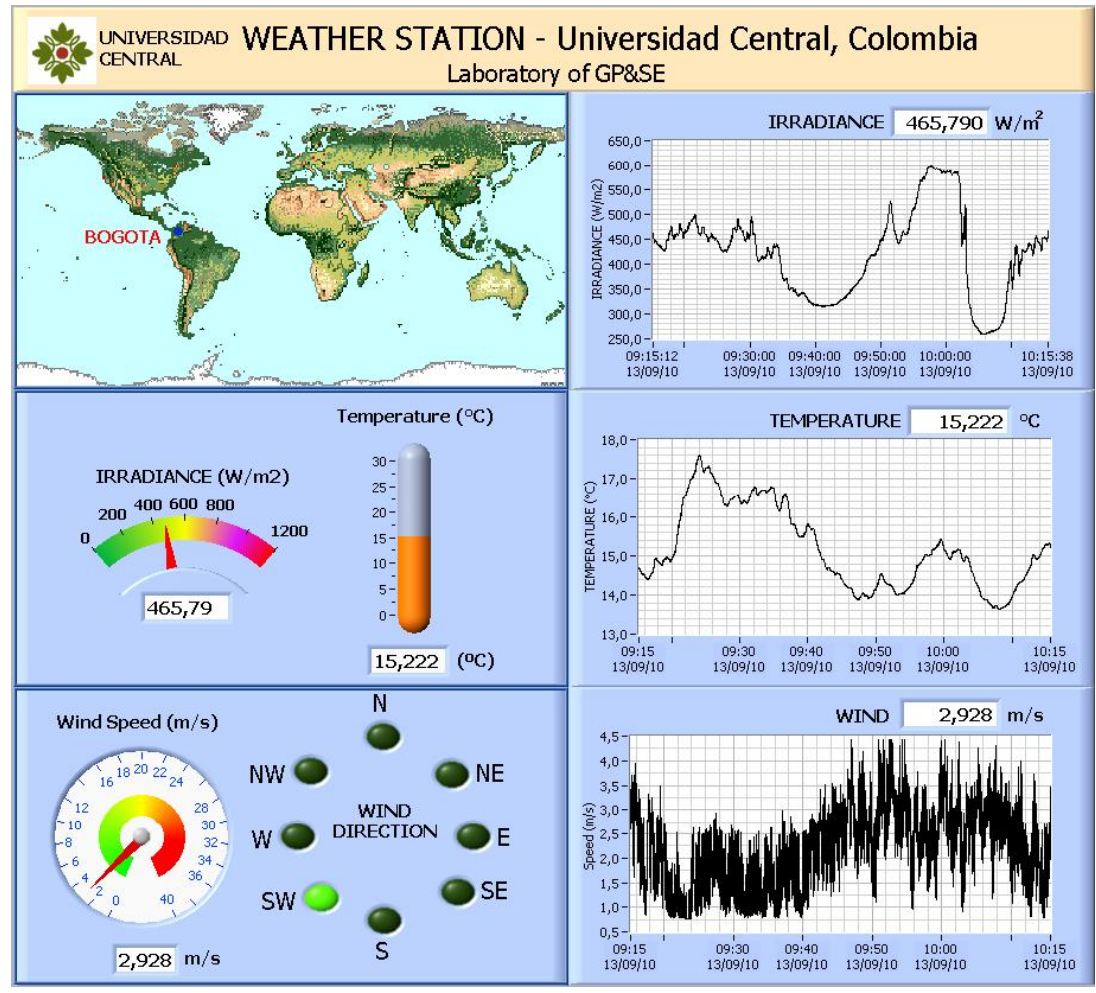

Figura 8. Panel frontal del VI para monitorear la temperatura ambiente, la radiación solar y la velocidad y dirección del viento.

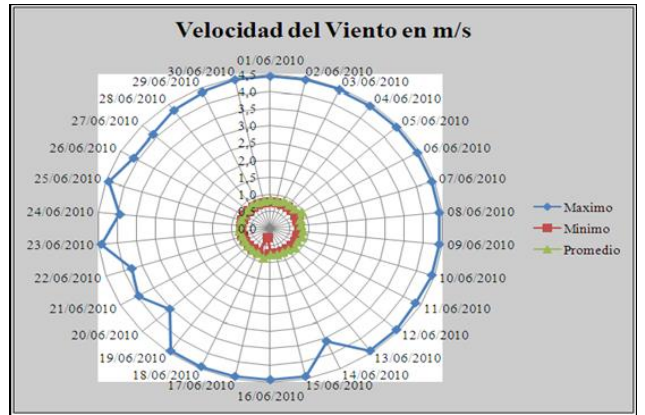

Figura 9. Perfil diario del caudal de aire para el mes de mayo de 2010. 


\begin{tabular}{cc}
\hline Parámetro & Valor \\
\hline Máximo & $4,4340 \mathrm{~m} / \mathrm{s}$ \\
\hline Mínimo & $0,2800 \mathrm{~m} / \mathrm{s}$ \\
\hline Promedio & $0,8212 \mathrm{~m} / \mathrm{s}$ \\
\hline
\end{tabular}

Cuadro 5. Valores de la velocidad del viento para junio de 2010.

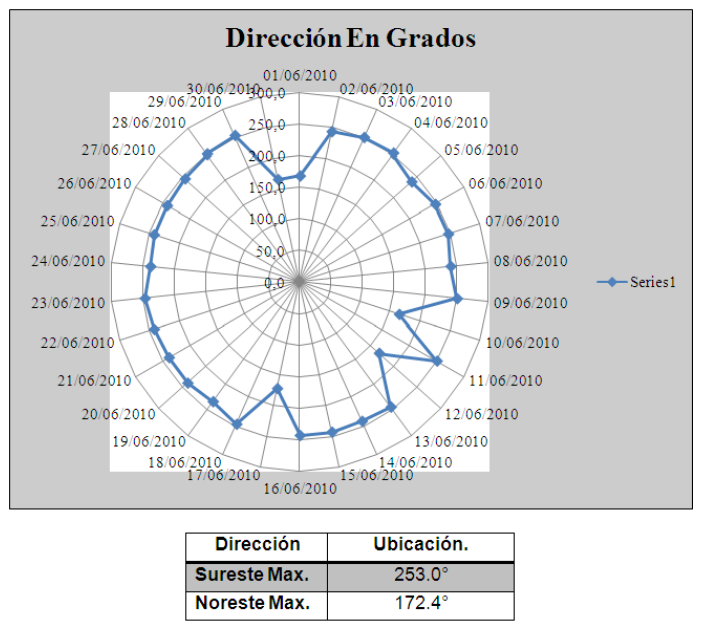

Figura 10. Comportamiento diario de la dirección del viento para el mes de junio de 2010 .

una incidencia general del 16,66\%. Esta magnitud indica que no solo se trata del microclima de la ciudad como se había pensado inicialmente y se debe al caudal asociado a la turbulencia de los edificios que rodean las instalaciones de la universidad, así como fenómenos naturales.

En el cuadro 7 se muestran los valores promedio diario-mensual de la radiación solar, temperatura ambiente y velocidad del viento durante los primeros doce meses de monitoreo de la estación.

\section{Conclusiones}

La estación meteorológica cumple con los requerimientos de diseño inicial al proporcionar mediciones bastante precisas de la temperatura, radiación global, velocidad y dirección del viento con posibilidad de almacenarlas en una computadora y realizar un tratamiento estadístico de datos. 


\begin{tabular}{|c|c|c|c|c|c|c|}
\hline & Vel & cidad ( & $\mathrm{m} / \mathrm{s})$ & Dirección (V) & & \\
\hline Fecha & Máximo & Mínimo & Promedio & Promedio & $\begin{array}{c}\text { Equivalente } \\
\text { en grados }\end{array}$ & Ubicación \\
\hline $01 / 06 / 10$ & 4,4340 & 0,7680 & 0,8396 & 6,0838 & 168,3 & NW \\
\hline $02 / 06 / 10$ & 4,4340 & 0,7670 & 0,7987 & 5,7090 & 242,4 & SW \\
\hline $03 / 06 / 10$ & 4,4340 & 0,7670 & 0,8620 & 5,8743 & 249,4 & SW \\
\hline $04 / 06 / 10$ & 4,4340 & 0,7670 & 0,8400 & 5,9193 & 251,3 & SW \\
\hline $05 / 06 / 10$ & 4,4340 & 0,7640 & 0,8424 & 5,5975 & 237,6 & SW \\
\hline $06 / 06 / 10$ & 4,4340 & 0,7650 & 0,9483 & 5,8200 & 247,1 & SW \\
\hline $07 / 06 / 10$ & 4,4340 & 0,7610 & 0,8171 & 5,8110 & 246,7 & SW \\
\hline $08 / 06 / 10$ & 4,4340 & 0,7610 & 0,8027 & 5,6443 & 239,6 & SW \\
\hline $09 / 06 / 10$ & 4,4340 & 0,7590 & 0,8512 & 5,8844 & 249,8 & SW \\
\hline $10 / 06 / 10$ & 4,4340 & 0,7580 & 0,8644 & 6,0039 & 164,9 & NW \\
\hline $11 / 06 / 10$ & 4,3710 & 0,7540 & 0,8047 & 5,8903 & 250,1 & SW \\
\hline $12 / 06 / 10$ & 4,4340 & 0,7520 & 0,8643 & 6,0852 & 168,3 & NW \\
\hline $13 / 06 / 2010$ & 4,4340 & 0,7520 & 0,7883 & 5,7640 & 244,7 & SW \\
\hline $14 / 06 / 2010$ & 3,6160 & 0,7520 & 0,7777 & 5,6846 & 241,3 & SW \\
\hline $15 / 06 / 2010$ & 4,4340 & 0,7520 & 0,7945 & 5,7240 & 243,0 & SW \\
\hline $16 / 06 / 2010$ & 4,4340 & 0,7520 & 0,7858 & 5,7248 & 243,0 & SW \\
\hline $17 / 06 / 2010$ & 4,4340 & 0,2800 & 0,9022 & 6,1805 & 172,4 & NW \\
\hline $18 / 06 / 2010$ & 4,4340 & 0,7650 & 0,8090 & 5,8091 & 246,6 & SW \\
\hline $19 / 06 / 2010$ & 4,4340 & 0,7650 & 0,8009 & 5,5146 & 234,1 & SW \\
\hline $20 / 06 / 2010$ & 3,5320 & 0,7680 & 0,7895 & 5,6321 & 239,1 & SW \\
\hline $21 / 06 / 2010$ & 3,9630 & 0,7680 & 0,7815 & 5,6341 & 239,2 & SW \\
\hline $22 / 06 / 2010$ & 3,8040 & 0,7660 & 0,7805 & 5,7139 & 242,6 & SW \\
\hline $23 / 06 / 2010$ & 4,4340 & 0,7650 & 0,8197 & 5,8118 & 246,7 & SW \\
\hline $24 / 06 / 2010$ & 3,9470 & 0,7640 & 0,7862 & 5,6001 & 237,7 & SW \\
\hline $25 / 06 / 2010$ & 4,4340 & 0,7650 & 0,8114 & 5,7197 & 242,8 & SW \\
\hline $26 / 06 / 2010$ & 4,1190 & 0,7655 & 0,7959 & 5,7168 & 242,7 & SW \\
\hline $27 / 06 / 2010$ & 4,1190 & 0,7655 & 0,8001 & 5,7629 & 244,6 & SW \\
\hline $28 / 06 / 2010$ & 4,2765 & 0,7618 & 0,8323 & 5,8834 & 249,8 & SW \\
\hline $29 / 06 / 2010$ & 4,3553 & 0,7639 & 0,8257 & 5,9596 & 253,0 & SW \\
\hline $30 / 06 / 2010$ & 4,4340 & 0,7660 & 0,8191 & 6,0357 & 166,2 & NW \\
\hline Promedio & 4,2928 & 0,7460 & 0,8212 & 5,8065 & 231,5 & \\
\hline
\end{tabular}

Cuadro 6. Datos de dirección y velocidad del viento para el mes de junio de 2010.

Se comprobó el funcionamiento y flexibilidad de los instrumentos virtuales desarrollados en el software de programación LabVIEW para la medición de variables físicas y almacenamiento de los datos obtenidos junto con un diseño óptimo de instrumentación electrónica; para otorgar mediciones fiables que podrían ser empleadas en el dimensionamiento de sistemas de generación limpia de energía eléctrica. 
Agradecimientos: este proyecto fue financiado por la Universidad Central y contó con el apoyo del Grupo de Materiales Semiconductores \& Energía Solar (GMS\&ES) de la Universidad Nacional de Colombia.

\begin{tabular}{lllll}
\hline & Mes & Rad. $\left(\mathbf{k W h} / \mathbf{m}^{2}\right)$ & Temp $\left({ }^{\circ} \mathbf{C}\right)$ & Viento $(\mathbf{m} / \mathbf{s})$ \\
\hline & Abril & 2,89 & 14,78 & 0,88 \\
\cline { 2 - 5 } & Mayo & 3,3 & 14,09 & 0,82 \\
\cline { 2 - 5 } & Junio & 3,73 & 14,6 & 0,82 \\
\cline { 2 - 5 } Año 2010 & Julio & 2,85 & 13,6 & 0,81 \\
\cline { 2 - 5 } & Agosto & 3,35 & 13,47 & 0,86 \\
\cline { 2 - 5 } & Sept. & 3,24 & 14,23 & 0,89 \\
\cline { 2 - 5 } & Oct. & 3,08 & 16,19 & 0,78 \\
\cline { 2 - 5 } Año 2011 & 2,47 & 15,31 & 0,92 \\
\cline { 2 - 5 } & Nov. & 2,68 & 14,82 & 0,85 \\
\cline { 2 - 5 } & Enero & 3,45 & 15,68 & 0,95 \\
\cline { 2 - 5 } & Febr. & 3,26 & 15,23 & 0,88 \\
\cline { 2 - 5 } & Marzo & 2,92 & 16,44 & 0,77 \\
\hline & Abril & 3,47 & 14,62 & 0,92 \\
\hline
\end{tabular}

Cuadro 7. Promedio mensual de las mediciones realizadas durante el primer año de operación.

\section{Referencias}

1. Mukaro, R., Carelse X.F., Olumekor, L.: First performance analysis of a siliconcell microcontroller based solar radiation monitoring system. Solar Energy No. 63 313-321. (1998)

2. Mukaro, R., Carelse X.F.: A microcontroller-based data acquisition system for solar radiation and environmental monitoring, IEEE Trans. Instrument. Meas, 48 1232-1238. (1999)

3. Wilson, A., Pearsal, N., Hill, R.: Installation and operation of the first city center PV monitoring station in the UK, Solar Energy, 59 19-26. (1997)

4. Benghanem M., Maafi, A.: Data acquisition system for photovoltaic systems performance monitoring. IEEE Trans. Instrument. Meas, 47 30-33. (1998)

5. Santos, A., Lopez, C., Garcia, A.: Automatic data acquisition System for PV solar Plant. Solar and Wind technology, Vol. 3, No. 4 259. (1986)

6. Lundqvist, C., Ossenbrink, H. A.: ESTI-LOG PV Plant Monitoring system, Solar energy Materials and Solar Cells, 47, 289-94. (1997)

7. NI CFP-2020.: Rugged Intelligent Ethernet Controller Interface for Compact FieldPoint with Removable Storage. [En línea]. http://sine.ni.com/nips/cds/ print/p/lang/en/nid/11572 USA (2010) 
8. NI CFP-AI-110.: Módulo de Entrada Analógica de Voltaje y Corriente con 8 Canales para Compact FieldPoint. [En línea]. http://sine.ni.com/nips/cds/ view/p/lang/es/nid/11581 USA (2010)

9. Forero, N.: Desarrollo de un prototipo de estación autónoma de medición automática de radiación solar velocidad de viento y temperatura ambiente. Tesis doctoral. Departamento de Física, Universidad Nacional de Colombia, Bogotá, Colombia, (2006)

10. Agencia Internacional de la Energía.: Photovoltaic power systems in selected IEA member countries: the second of a series of survey reports. Informe PVPS Ex.Co./TI 1997:1 (1997)

11. Kipp\&Zonen.: SP lite pyranometer. [En línea]. http://www.kippzonen.com/ (2009)

12. Borenstein, S.: The Market Value and Cost of Solar Photovoltaic Electricity Production, Center for the Study of Energy Markets (CSEM) Working Paper Series. (2008) 